\title{
Resolving the Atomic Structure of Materials Containing Light Elements by
}

\section{Annular-Bright-Field Electron Microscopy}

Lin $\mathrm{Gu}^{1}$, Dongdong Xiao ${ }^{1}$, and Yuichi Ikuhara ${ }^{2,3,4}$

1. Institute of Physics, Chinese Academy of Sciences/Beijing National Laboratory for Condensed, Matter Physics, Beijing, 100190, P.R. China

2. Institute of Engineering Innovation, The University of Tokyo, Tokyo, 113-8654, Japan

3. Nanostructures Research Laboratory, Japan Fine Ceramic Centre, Nagoya, 456-8587, Japan

4. WPI Advanced Institute for Materials Research, Tohoku University, Sendai, 980-8577, Japan

Unraveling the correlations between atomic structure and properties of materials requires resolving the locations and chemical types of all the atoms that make up the structure. Imaging using high-angle scattered electrons in aberration-corrected scanning transmission electron microscopy (STEM), also refers to high angle annular dark field imaging (HAADF), allows giving atomic resolution images that can be directly interpreted over a wide range of specimen thicknesses. However, the image contrast is strongly dependent on the atomic number Z, which makes it difficult to obtain information on the structural arrangement of the light elements in materials which contain the light and heavy elements at the same time ${ }^{[1,2]}$. Therefore, one effective strategy to enhance contrast from light atoms is to develop new imaging method which can weaken Z-dependence of image contrast so that light and heavy elements are visible simultaneously. The recently established annular bright-field imaging (ABF) using an annular detector located within a bright-field momentum range in aberration-corrected STEM has made direct imaging of light element come true, providing an unprecedented opportunity to reveal the configuration of light atoms which play a non-trivial role in relevant materials ${ }^{[3,4]}$.

Figure 1 shows the geometry of annular-bright-field imaging in aberration-corrected STEM, wherein an electron probe with convergent semi-angle $\alpha$ is focused to sub-angstrom dimension and scans across the specimen, and an ABF detector at the post column subtends a detecting angle range $\boldsymbol{q}$. The lithium and oxygen ions in $\mathrm{LiFePO}_{4}$, an important cathode for lithium ion battery, were clearly resolved, demonstrating the robustness of ABF imaging method. In this talk, we will present our recent efforts toward direct imaging of the lights atoms (e.g. $\mathrm{Li}, \mathrm{O}$, and $\mathrm{Na}$ ) in electrode materials for rechargeable batteries and probing their atomic-scale structure evolution under different electrochemical states ${ }^{[5-8]}$. 


\section{References:}

[1] N. D. Browning et al, Nature 366 (1993), 143.

[2] S. Hillyard et al, Ultramicroscopy 58 (1995), 6.

[3] E. Okunishi et al, Microsc. Microanal. 15 (2009), 164.

[4] S. D. Findlay et al, Appl. Phys. Lett. 95 (2009), 191913.

[5] Yang Sun et al, Nat. Commun. 4 (2013), 1870.

[6] Yong-Ning Zhou et al. Nat. Commun. 5 (2014), 5381.

[7] Haijun Yu et al, Angew. Chem. Int. Ed. 53 (2014), 8963.

[8] This work was supported by funding from the NSFC (11174334, 51325206) and the Chinese Academy of Sciences.

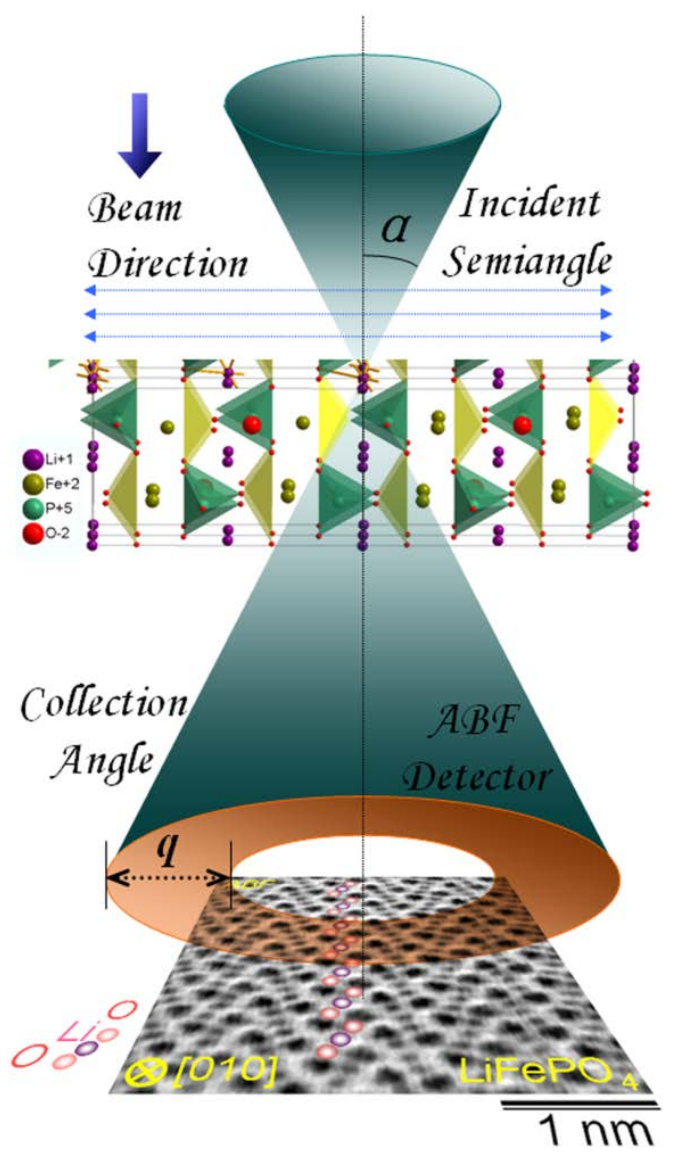

Figure 1. Schematic illustration of annular-bright-field imaging geometry. An electron probe with convergent semi-angle $\alpha$ is focused to sub-angstrom dimension and scans across the specimen. An ABF detector at the post column subtends a detecting angle range $\boldsymbol{q}$. A demonstration of lithium and oxygen sites within a $\mathrm{LiFePO}_{4}$ crystal along the [010] orientation is also shown. 